\title{
Observation of topical tacrolimus on high-risk penetrating keratoplasty patients: a randomized clinical trial study
}

\author{
Li-Ying Zhai ${ }^{1} \cdot$ Xiao-Rong Zhang $\mathbb{1}^{1,2} \cdot$ Huan $\mathrm{Liu}^{1} \cdot$ Yue Ma ${ }^{1} \cdot$ Hong-Chang $\mathrm{Xu}^{1}$
}

Received: 11 October 2018 / Revised: 14 May 2019 / Accepted: 22 August 2019 / Published online: 29 November 2019

(c) The Author(s), under exclusive licence to The Royal College of Ophthalmologists 2019

\begin{abstract}
Background/Objectives To evaluate the clinical efficacy of topical tacrolimus $0.1 \%$ and cyclosporine $1 \%$ on high-risk penetrating keratoplasty (PKP) patients.

Subjects/Methods A series of 49 high-risk PKP patients (49 eyes), 20 males, 29 females from the age of 4 months to 74 years of age with the mean of 32.5 from 2012 to 2017 were recruited in this study. The patients were randomly divided into two groups by receiving either topical tacrolimus $0.1 \%$ or cyclosporine $1 \%$ respectively. Twenty five patients were treated with topical tacrolimus $0.1 \%$ and 24 patients with topical cyclosporine $1 \%$. The traditional baseline management on these two groups were Tobramycin and Dexamethasone eye drops in the first 3 weeks and then tapered off. Clinical procedures and postoperative follow-up were documented.

Results After 6-54 months follow-up, with the average of 24 months, 11 of 24 high-risk patients (11 eyes) had graft rejection, the rejection rate was $45.8 \%$ in topical cyclosporine $1 \%$ group. The rejections occurred from 35 days to 20 months after PKP. Three patients had irreversible rejection. On topical tacrolimus $0.1 \%$ group, the rejection occurred in four patients (four eyes) with rejection rate of $16 \%$, and no irreversible rejection was observed. The graft rejection episodes were documented between 23 days and 24 months. As compared with the topical cyclosporine $1 \%$, topical tacrolimus $0.1 \%$, a key immunosuppressant, significantly decreased corneal graft rejection rate $(p=0.02)$.

Conclusions Topical tacrolimus $01 \%$ on high-risk PKP patients significantly prevented corneal graft rejection, and it had less adverse effects and was very safe to high-risk patients as to topical cyclosporine $1 \%$. Further case controlled randomized clinical trial studies are needed to establish the best management option for these high-risk patients.
\end{abstract}

\section{Introduction}

Corneal disorders are the most common causes of blindness world widely. Corneal transplantation currently is the most frequently performed and the best way to prevent the corneal blindness. Despite immune privilege status of the human cornea, immune-mediated graft rejection remains the leading cause of corneal graft failure, particularly in the high-risk graft recipients and/or with contraindication to corticosteroid

Xiao-Rong Zhang

zxr1110@sina.com

1 Division of Ocular Injuries, Department of Ophthalmology, The Third Affiliated Hospital of Hebei Medical University, Shijiazhuang 050051 Hebei, China

2 Hebei OPO Eye Bank, The Third Affiliated Hospital of Hebei Medical University, Shijiazhuang 050051 Hebei, China therapy. The rate of graft rejection was previously reported as high as between 60 and 90\% depending on the criteria used to classify high risk even with the administrating of immunosuppressive agents [1]. Patients were defined as high risk as having a history of previous graft rejection failed, chemical injury, herpes simplex virus keratitis (HSVK), large grafts, younger recipient age, glaucoma,and at least two quadrants of stromal neovascularization. [1-3]. To increase the success rate of high-risk corneal graft rejection a number of strategies had been employed including both local and systemic immunosuppressants. Even corticosteroids and cyclosporine A are the two top selected immunosuppressive agents, but high rejection rate and enormous side effects were documented such as secondary glaucoma, steroid cataract, corneal epithelium defects, delayed wound healing and local irritable.

Several studies have shown that graft survival rates were enhanced by using systemic and topical immunosuppression of cyclosporine and/or tacrolimus [1, 4, 5]. Tacrolimus 
(FK506) is well known as an immunosuppressive agent in corneal graft rejection, which exhibits similar pharmaceutical role as cyclosporine A via inhibiting cellular toxins of $\mathrm{T}$ cells and mixed lymph cells. As a newly safety and highly efficacy agent, it shows 10-100 folds of immunosuppression as to cyclosporine A [6]. Owing to its less toxic and side effects, it is also used in patients with vernal keratoconjunctivitis and Mooren's ulcer and systemic cancer patients. Here we reported the results of topical tacrolimus $0.1 \%$ as compared with topical cyclosporine $1 \%$ on high-risk corneal transplantation patients in a randomized clinical trial study.

\section{Subjects and methods}

A total of 49 patients, 20 males and 29 females, with high-risk penetrating keratoplasty (PKP) were randomly enroled in this study over a period of 6 years from 2012 to 2017 in consecutive series. Twenty five patients (25 eyes) were managed by topical tacrolimus $0.1 \%$, while 24 patients ( 24 eyes) were treated with topical cyclosporine $1 \%$. The diagnosis and clinical details of patients were documented. According to all the risk factors, a high-risk scoring system was adapted based on published preoperative risk factors for immune corneal graft rejection (Table 1) [4]. This study was approved by the institutional review board. All patients completed clinical trial registration and the study was adhered to the tenets of the Declaration of Helsinki. Informed consent was obtained from each patient after explanation of the nature and possible consequences of the study.

All the corneal transplantations were performed by one and/or two of the authors. PKP were performed via standard procedure, and combined cataract extraction with IOL implantation were done on six patients. All the corneal grafts were obtained from local donors within $8 \mathrm{~h}$ postmortem and kept at $4{ }^{\circ} \mathrm{C}$ in Optisol-GS corneal storage media (Bausch \& Lomb, Rochester, NY, US). The corneal transplantation was done within 1 week after punched out.

All patients on the first 3 weeks received traditional treatment with Tobramycin Dexamethasone eye ointment (Alcon, Novartis, USA) once every night (QN), and

Table 1 Preoperative risk scoring for high-risk patients taking corneal transplantation

\begin{tabular}{ll}
\hline Patients high-risk characteristics & Risk scores \\
\hline Each quadrant of stromal vascularization & 1 \\
Each quadrant of peripheral anterior synechiae & 1 \\
Preoperative glaucoma & 1 \\
Each previous graft & 1 \\
Herpes simplex virus keratitis & 2 \\
Alkali burns & 4 \\
\hline
\end{tabular}

Tobramycin Dexamethasone eye drops (Alcon, Novartis, USA) every $2 \mathrm{~h} /$ day, and tapered off to $\mathrm{q} 4 \mathrm{~h}$ and discontinued at the end of third week, from the second week starting topical tacrolimus $0.1 \%$ (Senju Pharmaceutical Co, Japan), and cyclosporine $1 \%$ (North China Pharmaceutical Co, China) in each group for 18 months postoperatively. Corneal graft rejection was defined as the presence of sudden decrease of visual acuity, graft oedema, endothelial rejection lines, keratic precipitate and the formation of neovascularization. In patients who experienced graft rejections during management, the doses of tobramycin and dexamethasone eye drops and tacrolimus or cyclosporine were increased and continued for a period of 1 year after rejection. Tobramycin and dexamethasone eye drops would be tapered off as early as possible if graft was stable to prevent its adverse effects. When the graft rejection was controlled then tapered off tacrolimus or cyclosporine. The time to graft rejection and the number of episodes were documented. The follow up was considered as ending point of this study, graft failure, death. Persistent graft oedema and cloudiness with loss of transparency after 2 months of rejection treatment was considered as irreversible rejection. And re-graft was considered.

All patients were followed up daily in the first week, and once a week in the first month, and then every month. At each visit, slit lamp examination, Snellen visual acuity, intraocular pressure, corneal clarity, neovascularization, state of sutures and any clinical evidence of rejection were all documented.

Statistical analyses were performed on computer (SPSS, ver. 6.1; SPSS Science, Chicago, IL). The $t$ test was used to evaluate the comparability of the preoperative risk scoring for patients in two groups. The data for evaluation of two medications of immunosuppressive activity were compared via using the $\chi^{2}$ test. $p<0.05$ was considered significant.

\section{Results}

We recruited 49 consecutive high-risk patients (49 eyes) who underwent PKP, and six of them combined cataract extraction with IOL implantation. The patient clinical data and diagnosis are summarized in Tables 2 and 3. Patients who were classified as high-risk ones were divided into two groups, tacrolimus group and cyclosporine group. The mean follow-up of the topical $0.1 \%$ group was $24.3 \pm 8.9(\mathrm{M} \pm$ SD) months, while the topical cyclosporine $1 \%$ group was $23.6 \pm 10.4(\mathrm{M} \pm \mathrm{SD})$ months. The preoperative risk scorings in tacrolimus and cyclosporine group were $3.46 \pm 0.24$ $(\mathrm{M} \pm \mathrm{SD})$ and $3.79 \pm 0.28(\mathrm{M} \pm \mathrm{SD})$, there was no clinical statistic significant difference $(p=0.166)$ (Fig. 1).

Of all the 49 patients (49 eyes), 33 patients (33 eyes) (77.78\%) had enhanced best corrected visual acuity to the range of hand motion to 0.6 after $\mathrm{PKP}$, and 10 patients 
Table 2 Diagnosis and risk factors of high-risk patients

\begin{tabular}{lll}
\hline Diagnosis & Number of patients & Percentage $(\%)$ \\
\hline Chemical corneal injury & 10 & 20.4 \\
Herpes simplex virus keratitis & 7 & 14.29 \\
Corneal scar & 9 & 18.37 \\
Children & 4 & 8.16 \\
Corneal dystrophy & 12 & 24.49 \\
Others w/neovascularization & 7 & 14.29
\end{tabular}

Table 3 Clinical data of all patients

\begin{tabular}{llll}
\hline & $\leq 20$ & $20-50$ & $\geq 50$ \\
\hline Age (years) & 15 & 16 & 18 \\
Gender & & & \\
Male & 8 & 9 & 10 \\
Female & 7 & 7 & 8 \\
Risk factors & & & \\
HSVK & 2 & 4 & 5 \\
Chemical burns & 3 & 5 & 5 \\
Previous failed grafts & 4 & 3 & 3 \\
Younger recipient age & 4 & 0 & 0 \\
$\geq 2$ quadrants of stromal vascularization & 2 & 4 & 5 \\
\hline
\end{tabular}

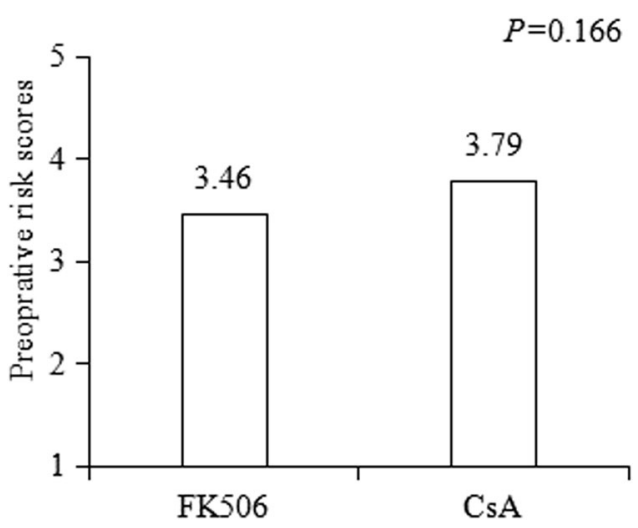

Fig. 1 Comparison of the preoperative risk scores between FK506 and CsA group

(10 eyes) $(22.22 \%)$ had not changed their visual acuity in these two groups. Four younger children were excluded from the analysis because they were not able to take the Snellen visual acuity test. Twenty-one patients $(91.3 \%)$ had increased visual acuity in tacrolimus group, and 14 patients $(63.64 \%)$ had enhanced visual acuity in cyclosporine group (Table 4), significant statistic difference was noted $\left(\chi^{2}=\right.$ 4.98, $p=0.035$ ).

Figure 2 showed slit lamp photographs of anterior views of two typical high-risk patients pre- and postoperatively managed by topical tacrolimus $0.1 \%$ (a and $\mathbf{b}$ ). These two patients had different preoperative risk scorings. Case one

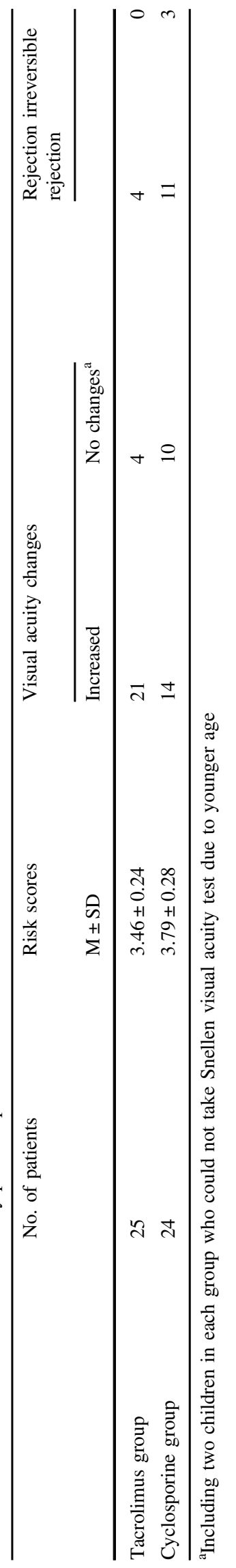



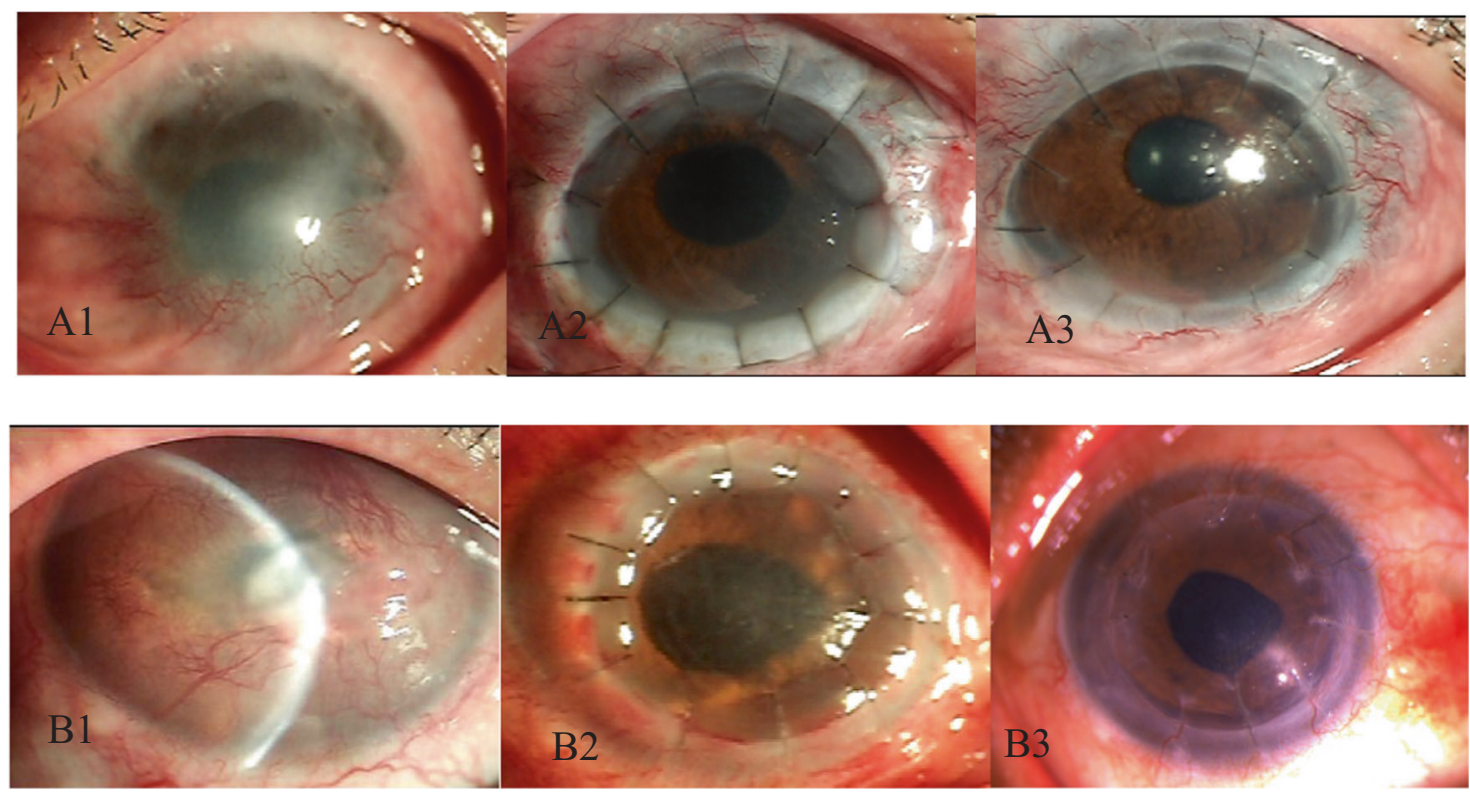

Fig. 2 Slit lamp photographs of Anterior views of two typical high-risk patients pre- and postoperatively managed by topical tacrolimus. Case one (a) from a 13-year-old boy who had chemical burns on his left eye and previous five times PKPs admitted to the hospital (A1) and 1 week after PKP (A2) and 36 months (A3). Case two (b) a 52-year-old man

(a) was a 13-year-old boy who had chemical burns on his left eye and previous five times PKPs in local hospital and transferred to our clinic. PKP was performed and topical tacrolimus $0.1 \%$ was used, photographs showed the patient was admitted to the hospital (top left) and 1 week after PKP (top middle) and 36 months (top right). Case two (b) was a 52-year-old man with right eye corneal foreign body secondary bacterial infection with neovascularization (bottom left) and referred to our clinic. He underwent PKP after managing the infection and neovascularization and his graft remained stable on topical tacrolimus $0.1 \% 1$ week (bottom middle) and 18 months (bottom right), respectively.

On tacrolimus group of 25 patients (25 eyes), four patients (16\%) experienced episodes of graft rejection, of whom two had multiple episodes. The rejection episodes occurred between 23 days and 24 months. The graft rejections were controlled with intensive topical tacrolimus and prednisolone. No irreversible graft rejection was observed in tacrolimus group. Figure 3 demonstrated a 59-year-old man had secondary glaucoma 1 week after PKP and reversable immune graft rejection 6 months after PKP and successfully controlled by conventional treatment. During follow-up of 24 patients in cyclosporine group, 11 patients (11 eyes) $(45.8 \%)$ had graft rejections, six of them had multiple graft rejections, three patients had irreversible rejection resulting in graft failure and treated with PKP again. The rejections were documented between 35 days and 20 months. Kaplan-Meier curve was drawing according to the occurring of rejections during the time frame of with right eye corneal foreign body secondary bacterial infection with neovascularization (B1). He underwent PKP after managing the infection and neovascularization and his graft remained stable on one week (B2) and 18 months (B3), respectively

follow-up (Fig. 4). Topical tacrolimus $0.1 \%$ was effective in preserve the graft survival in patients with high-risk PKP as compared with topical cyclosporine $1 \%$, it has clinically significant difference $\left(\chi^{2}=4.63, p=0.02\right)$. There was no significant difference in IOP among the two groups in follow-up. We have not observed any serious adverse effects on tacrolimus group in this study. The complications of PKP were observed in several patients on both groups, such as secondary glaucoma, infection and neovascularization, they were successfully treated accordingly. All the patients preserved the eyeballs during the period of followup. No patients dropped off from the study.

\section{Discussion}

Corneal disorders have been the major causes of blindness currently. As the most successful tissue and/or organ transplantation, corneal transplantation has been performed to control the corneal blindness and to restore the patient's visual acuity world widely. But allograft rejection remains the most common cause for corneal graft failure, particularly in high-risk patients. Studies reported that the high-risk corneal transplantation, in which the graft had a 5-year survival rate below $35 \%$ with graft rejection [7-9]. Corneal graft rejection is a T-cell-mediated immune response [10]. Systemic and topical tacrolimus and/or cyclosporine are effective in reducing rejection rates in high-risk grafts, which have been shown in animal models and humans 


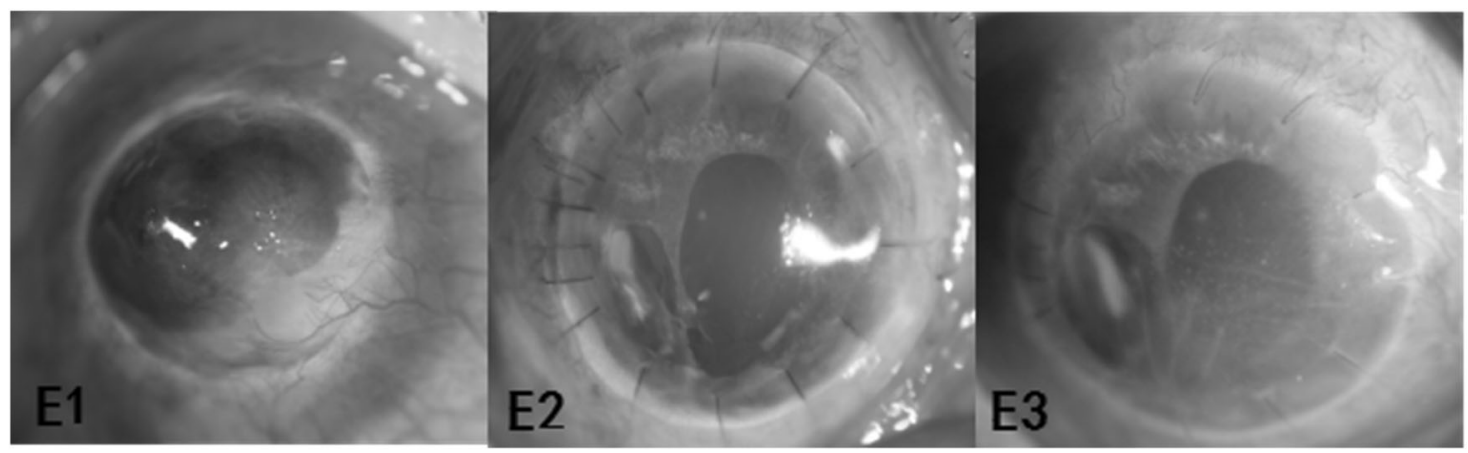

Fig. 3 Slit lamp photographs of anterior views of a 59-year-old man from FK506 group experienced of immune graft rejection 6 months after PKP. A 59-year-old Chinese man with right eye corneal posterior elastic membrane ectasia underwent PKP, photograph was taken

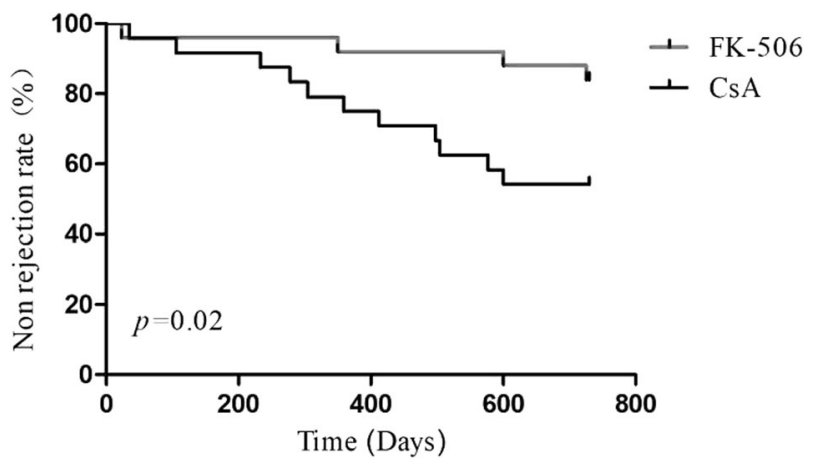

Fig. 4 Kaplan-Meier curve of immune corneal graft rejections in topical FK506 and CsA group after PKP

$[3,11]$. Tacrolimus was first discovered in 1984, which is now utilized as an immunosuppressant in organ and/or tissue transplantation. Tacrolimus and cyclosporine (CsA) have similar action by binding to the cytoplasmic FK506binding protein (FKBP-12) called immunophilins to inhibit the $\mathrm{Ca} 2+$ dependent diphosphatase activity of calcineurin $(\mathrm{CaN})$ that inhibits clonal expansion of $\mathrm{T}$ lymphocytes. But tacrolimus has more 10-100 folds potent immunosuppression than cyclosporine and less side effects [12-16].

Tacrolimus is a macrolide isolated from the fermentation broth of a strain of soil fungus, Streptomyces tsukubaensis found in Tsukuba area in Japan. Previous studies have demonstrated that tacrolimus is highly lipophilic, by using a carrier of liposome to make liposomal and/or oil formulations, it showed relatively great corneal penetration and distribution in ocular tissues $[12,17]$. Concentration of the drug in anterior chamber reached its peak at $1 \mathrm{~h}$ after topical tacrolimus $0.05 \%$.

Kobayashi first reported that tacrolimus was a new immunosuppressive agent to inhibit corneal graft rejection in rabbit eyes in 1989 [18]. Animal studies showed that tacrolimus had very effective immunosuppression action and it could inhibit corneal neovascularization, corneal oedema, cloudiness and pseudo-pterygium and to improve before surgery (E1). One week after PKP he developed secondary glaucoma, but the graft remained transparency (E2). The immune reversable graft rejection was noted in 6 months after PKP (E3), it was successfully controlled with conventional therapy

the graft's survival rates and to decrease the graft rejection rates in experimental corneal transplantation $[19,20]$. Recent clinical studies demonstrated that topical and systemic administration of tacrolimus was safe and effective in reducing graft rejection and prolonging graft survival in humans [5, 6, 21], the rate of graft transparency and visual acuity were all better than cyclosporine after PKP.

Zhang et al. reported that topical tacrolimus $0.1 \%$ suppressed graft rejection in high-risk corneal transplantation patients in 1-year follow-up [22]. The rejection rate was significantly lower in tacrolimus group than in cyclosporine group $(p<0.05)$. Dhaliwal et al. reported that all the highrisk patients did not experience irreversible graft rejection treated with topical tacrolimus after transplantation [11]. In our study, there was no irreversible graft rejection occurred in topical tacrolimus group as well. Graft rejection correlated significantly with the preoperative risk scores.

Joseph observed that no patients who were followed for over 30 months developed a graft rejection, suggesting that the risk of graft rejection in these high-risk patients is greatest in the first 30 months [15]. We had the identical result that no patients developed graft rejection after 30 months follow-up in our study. Investigators reported that nonimmune-mediated graft failure had occurred over 31 months after PKP [17]. The major factors responsible for nonimmune rejection were raised intraocular pressure, bacterial keratitis and stem cell failure.

Host corneal neovascularization is a main cause and risk factor for impaired vision and is the key to damage the cornea immune privilege status and to lead the graft rejection even failure in PKP patients [23]. The extent of corneal neovascularization correlates with the risk of graft rejection, more corneal neovascularization was closely correlated to earlier graft rejection, and poorer progression in these patients. Several studies have implicated that the involvement of deeper corneal layers by neovascularization is important in inducing an immune alloantigenic response $[2,24,25]$. It is well known that the number of corneal 
quadrants invaded by vessels as well as the number of vessels in each quadrant increase the risk of rejection [26]. Khodadoust et al. defined the rejection risks based on the degree of host bed vascularization: avascular, mild (1-3 vessels), moderate (4-10 vessels) and heavy (>10 vessels). They have noted that, in heavily vascularized eyes, $65 \%$ of grafts started to reject, and all grafts finally failed despite strong immunosuppressive treatment [27].

Research works have showed that tacrolimus may inhibit the inflammation induced by fungi and alleviate the severity of corneal damage at an early stage of fungi keratitis. It combined with low dose of glucocorticoids that can prevent the early immunological rejection after PKP for fungal keratitis effectively and safely [28]. $\mathrm{CaN}$ is a main modulating factor on the pathway of cellular immune system, fungus has $\mathrm{CaN}$, which plays an important role in signalling activity and fungal pathogen, further leads to inflammation. Tacrolimus is a CaN inhibitor, which combines antifungal treatments clinically to manage fungal infections, to prevent graft rejections in PKP patients.

Rejection episode signs were observed by sudden cornea oedema with epithelial or endothelial line, subepithelial infiltrates, anterior chamber reaction or keratic precipitates. Neovascularization, also a sign of graft rejection, is a complex, unclear process, involving interleukins, vascular endothelial growth factor and a variety of immune cells [11]. Graft rejection correlated significantly with the preoperative risk scores [15]. Higher risk score was closely correlated with the occurrence of graft rejection and even failure, which suggested that it was very useful to high-risk score patients and planed immunosuppressive strategies accordingly.

Re-grafts damaged the normal physiologic mechanism of the human eyes, increased the number and variety of antigens and further enhanced the rejection rate. Hence the re-grafts in some ways were to preserve the eyeball, not always to increase the patient's visual acuity. We reported four graft rejections in tacrolimus group, and 11 rejections in cyclosporine group. They all underwent re-grafts, one child with alkali burns had received re-graft five times (Fig. 2a) in tacrolimus group, and successfully restored the eyeball and clear cornea. In cyclosporine group, three patients (three eyes) underwent multiple re-grafts because of irreversible rejection.

Corneal transplantation for HSVK is associated with increased risk of failure owing to rejection or recurrence of infection $[1,29]$. Herpes simplex virus has a natural ability to establish life-long latency, reactivation of latent infection in the trigeminal ganglion and/or the cornea itself leads to recurrent disease. In one study, Cobo et al. reported rejection to be the principal cause of graft failure in 64\% [30]. Australian Corneal Graft Registry study indicating that recurrence free grafts have high survival rates suggest that if routine antiviral prophylaxis following transplantation reduces rates of recurrence, it is likely to enhance graft survival [26]. Tacrolimus, like any immunosuppressive agent to prevent graft rejection, could cause a recurrence of a pre-existing infection of HSVK. Precaution should be given to these patients.

Children are high-risk populations for corneal transplantation. Corneal disorders in children are worse than in adults, cornea cloudiness will affect the growth of their visual function, and lead to amblyopia in children. Studies have shown that an increased risk of rejection was seen when the transplantation was performed in a young host bed $[31,32]$. This is due to a combination of a robust immune response and also due to difficulty in recognizing these episodes early due to inability to perform accurate examinations on children in the office. Corneal transplantation should be performed earlier to reduce the period of visual deprivation in these populations.

Although the mechanism of action of tacrolimus is similar to that of cyclosporine, but cyclosporine and tacrolimus bind different intercellular proteins. Cyclosporine binds cyclophilin and tacrolimus binds FKBP-12 [33]. Tacrolimus has been reported to have fewer systemic side effects than CsA, even given that its immunosuppressive effect is 25-100 times more powerful than CsA [34, 35]. It has been used in various studies to prevent graft rejection in high-risk patients. Two to twelve milligrams per day of systemic tacrolimus has been shown to reduce graft rejection in high-risk corneal transplantation, with a graft survival of $65 \%$ [1]. The therapeutic target range of $1-12 \mathrm{mg} / \mathrm{L}$ was achieved with a mean daily dose of $4.4 \mathrm{mg}$. However, the optimum length of treatment is still not known [4]. In another study, Joseph et al. found that only eight of the 43 corneal transplant patients who used tacrolimus prophylactically ( $1 \mathrm{mg}$ twice daily) had rejection episodes, and five of these experienced rejections related graft failure [1]. These patients might have benefited from higher doses of tacrolimus or a combination treatment with another immunosuppressive agent. Study had demonstrated that systemic tacrolimus decreased graft rejection rates in high-risk PKP after graft failure with systemic cyclosporine [17]. In a prospective study, Yamazoe et al. showed that treatment with tacrolimus $(10-20 \mathrm{ng} / \mathrm{mL})$ resulted in significantly fewer graft rejection episodes and longer graft survival than CsA, probably due to its more effective suppression of alloimmunity [21]. Moreover, patients treated with tacrolimus tolerated the drug better than those treated with CsA; however, 20\% discontinued tacrolimus treatment because of renal dysfunction and muscle pain, which might also be related to previous CsA treatment they had received. Hypertension (23\%) is the most common side effect of systemic tacrolimus treatment, followed by headaches, malaise and gastrointestinal upset [1]. Systemic side effects 
have forced the patients to discontinue the medication and dropped off from the study. It was recommended that systemic tacrolimus should be used for short periods, and it should be avoided in patients with compromised immune systems. In such hosts topical tacrolimus administration should be preferred. Few case series have reported beneficial effect of topical tacrolimus in human high-risk corneal transplantation. Dhaliwal et al. [6] used off-label topical tacrolimus $0.03 \%$ ointment in four patients with high-risk corneal transplantation and did not have any rejection episode. Tacrolimus eye drops as adjunctive therapy, it may be able to play an important role in patients with severe corneal endothelial rejection refractory to conventional steroid treatment [36]. In addition, Magalhaes et al. [12] retrospectively compared the combination of topical prednisolone $1 \%$ and tacrolimus $0.03 \%$ eye drops to prednisolone eye drops alone in high-risk corneal transplantation. They noted a significantly lower rate of irreversible graft rejections in the prednisolone plus tacrolimus group than in the prednisolone-alone group without increasing IOP. Topical tacrolimus may be an excellent way to treat high-risk patients with advanced glaucoma because postoperative uncontrolled IOP is a risk factor for graft failure $[1,4]$. For topical application, it was safe, and we did not observe any serious adverse effects in high-risk patients in this study. Topical administration of tacrolimus might be desirable to avoid its systemic adverse effects.

This study is limited by its small sample size and heterogeneous aetiologies even the preoperative high-risk scores are not significant differences in the two groups. The optimal treatment duration of topical tacrolimus is still not known.

\section{Conclusions}

In our study, we found that topical tacrolimus $0.1 \%$ was effective in reducing the risk of corneal graft rejection in high-risk patients. In conclusion, on the traditional baseline management with corticosteroids, topical tacrolimus $0.1 \%$ on high-risk corneal transplantation patients significantly decreased corneal graft rejection, and it had less adverse effects and was very safe to high-risk patients as to topical cyclosporine $1 \%$. Further case controlled randomized clinical trial studies are needed to establish the best management option for these high-risk patients.

\section{Summary}

\section{What was known before}

- To evaluate the clinical efficacy of topical tacrolimus $0.1 \%$ and cyclosporine $1 \%$ on high-risk PKP patients.

\section{What this study adds}

- Topical tacrolimus $01 \%$ on high-risk PKP patients significantly prevented corneal graft rejection, and it had less adverse effects and was very safe to high-risk patients as to topical cyclosporine $1 \%$.

Funding This work was supported by the Bureau of Hebei Science Technology [grant number 182777112D], Shijiazhuang, Hebei, China.

\section{Compliance with ethical standards}

Conflict of interest The authors declare that they have no conflict of interest.

Publisher's note Springer Nature remains neutral with regard to jurisdictional claims in published maps and institutional affiliations.

\section{References}

1. Joseph A, Raj D, Shanmuganathan V, Powell RJ, Dua HS. Tacrolimus immunosuppression in high-risk corneal grafts. Br J Ophthalmol. 2007;91:51-5.

2. Maguire MG, Stark WJ, Gottsch JD, Stulting RD, Sugar A, Fink $\mathrm{NE}$, et al. Risk factors for corneal graft failure and rejection in the collaborative corneal transplantation studies. Collaborative Corneal Transplantation Studies Research Group. Ophthalmology. 1994;101:1536-47.

3. Hill JC. Systemic cyclosporine in high-risk keratoplasty. Short versus long-term therapy. Ophthalmology. 1994;101:128-33.

4. Sloper CM, Powell RJ, Dua HS. Tacrolimus (FK506) in the management of high-risk corneal and limbal grafts. Ophthalmology. 2001;108:1838-44.

5. Magalhaes OA, Marinho DR, Kwitko S. Topical $0.03 \%$ tacrolimus preventing rejection in high-risk corneal transplantation: a cohort study. Br J Ophthalmol. 2013;97:1395-8.

6. Dhaliwal JS, Mason BF, Kaufman SC. Long-term use of topical tacrolimus (FK506) in high-risk penetrating keratoplasty. Cornea. 2008;27:488-93.

7. Dana MR, Qian Y, Hamrah P. Twenty-five-year panorama of corneal immunology: Emerging concepts in the immunopathogenesis of microbial keratitis, peripheral ulcerative keratitis, and corneal transplant rejection. Cornea. 2000;19:625-643.

8. Lam H, Dana MR. Corneal graft rejection. Int Ophthalmol Clin. 2009;49:31-41.

9. Zhu SN, Yamada J, Streilein JW, Dana MR. ICAM-1 deficiency suppresses host allosensitization and rejection of MHC-disparate corneal transplants. Transplantation. 2000;69:1008-1013.

10. Boisgerault F, Liu Y, Anosova N, Ehrlich E, Dana MR, Benichou G. Role of CD4+ and CD8 + T cells in allorecognition: lessons from corneal transplantation. J Immunol. 2001;167: 1891-9.

11. Hill JC, Maske R. An animal model for corneal graft rejection in high-risk keratoplasty. Transplantation. 1988;46:26-30.

12. Yuan J, Zhai JJ, Huang X, Zhou SY, Chen JQ. Ocular safety and pharmacokinetics study of FK506 suspension eye drops after corneal transplantation. J Ocul Pharm Ther. 2012;28:153-8.

13. Coster DJ, Williams KA. The impact of corneal allograft rejection on the long-term outcome of corneal transplantation. Am J Ophthalmol. 2005; 140:1112-22. 
14. Griffith BP, Bando K, Hardesty RL, Armitage JM, Keenan RJ, Pham SM, et al. A prospective randomized trial of FK506 versus cyclosporine after human pulmonary transplantation. Transplantation. 1994;57:848-51.

15. Tamura K, Fujimura T, Iwasaki K, Sakuma S, Fujitsu T, Nakamura K, et al. Interaction of tacrolimus (FK506) and its metabolites with FKBP and calcineurin. Biochem Biophys Res Commun. 1994;202:437-43.

16. Du S, Hiramatsu N, Hayakawa K, Kasai A, Okamura M, Huang T, et al. Suppression of NF-kappa B by cyclosporin a and tacrolimus (FK506) via induction of the C/EBP family: implication for unfolded protein response. J Immunol. 2009;182:7201-11.

17. Pleyer U, Lutz S, Jusko WJ, Nguyen KD, Narawane M, Ruckert D, et al. Ocular absorption of topically applied FK506 from liposomal and oil formulations in the rabbit eye. Investig Ophthalmol Vis Sci. 1993;34:2737-42.

18. Kobayashi C, Kanai A, Nakajima A, Okumura K. Suppression of corneal graft rejection in rabbits by a new immunosuppressive agent, FK-506. Transpl Proc. 1989;21:3156-8.

19. Mills RA, Jones DB, Winkler CR, Wallace GW, Wilhelmus KR. Topical FK-56 prevents experimental corneal allograft rejection. Cornea. 1995;14:157-60.

20. Reinhard T, Mayweg S, Reis A, Sundmacher R. Topical FK506 as immunoprophylaxis after allogeneic penetrating normal-risk keratoplasty: a randomized clinical pilot study. Transpl Int. 2005;18:193-7.

21. Yamazoe K, Yamaguchi T, Omoto M, Shimazaki J. Efficacy and safety of systemic tacrolimus in high-risk penetrating keratoplasty after graft failure with systemic cyclosporine. Cornea. 2014;33:1157-63.

22. Zhang L, Li S, Zhang N, Bian J, Shi WY. The immunosuppressive activity of tacrolimus eye drops in high-risk corneal transplantation. J Clin Ophthalmol. 2017;25:193-6.

23. Bachmann B, Taylor RS, Cursiefen C. Corneal neovascularization as a risk factor for graft failure and rejection after keratoplasty. Ophthalmology. 2010;117:1300-5.

24. Williams KA, Roder D, Esterman A, Muehlberg SM, Coster DJ. Factors predictive of corneal graft survival. Report from the Australian Corneal Graft Registry. Ophthalmology. 1992;99:403-414.
25. Kamp MT, Fink NE, Enger C, Maguire MG, Stark WJ, Stulting RD. Patient-reported symptoms associated with graft reactions in high-risk patients in the collaborative corneal transplantation studies. Collaborative Corneal Transplantation Studies Research Group. Cornea. 1995;14:43-48.

26. The Australian Corneal Graft Registry. 1990 to 1992 report. Aust N. Z J Ophthalmol. 1993;21 2 Suppl :1-48.

27. Khodadoust AA, Silverstein AM. Studies on the nature of the privilege enjoyed by corneal allografts. Investig Ophthalmol. 1972;11:137-48.

28. Xiang DM, Wang YX, Jia YN, Li SX, Zhai HL, Shi WY, et al. The observation of tacrolimus eye drops preventing the early immunological rejection after penetrating keratoplasty for fungal keratitis. Chin J Ophthalmol. 2017;53:305-10.

29. Larkin DF. Corneal transplantation for herpes simplex keratitis. $\mathrm{Br}$ J Ophthalmol. 1998;82:107-8.

30. Cobo LM, Coster DJ, Rice NS, Jones BR. Prognosis and management of corneal transplantation for herpetic keratitis. Arch Ophthalmol. 1980;98:1755-9.

31. Musch DC, Meyer RF. Risk of endothelial rejection after bilateral penetrating keratoplasty. Ophthalmology. 1989;96:1139-43.

32. Sanfilippo F, MacQueen JM, Vaughn WK, Foulks GN. Reduced graft rejection with good HLA-A and B matching in high-risk corneal transplantation. N. Engl J Med. 1986;315: 29-35.

33. Siekierka JJ, Sigal NH. FK-506 and cyclosporin A: immunosuppressive mechanism of action and beyond. Curr Opin Immunol. 1992;4:548-52.

34. Geba GP, Ptak W, Askenase PW. Topical tacrolimus and cyclosporin A differentially inhibit early and late effector phases of cutaneous delayed-type and immunoglobulin E hypersensitivity. Immunology. 2001;104:235-42.

35. Sawada S, Suzuki G, Kawase Y, Takaku F. Novel immunosuppressive agent, FK506. In vitro effects on the cloned T cell activation. J Immunol. 1987;139:1797-803.

36. Ghaffari R, Ghassemi H, Zarei-Ghanavati M, Latifi G, Dehghani $\mathrm{S}$, Haq Z, et al. Tacrolimus eye drops as adjunct therapy in severe corneal endothelial rejection refractory to corticosteroids. Cornea. 2017;36:1195-9. 